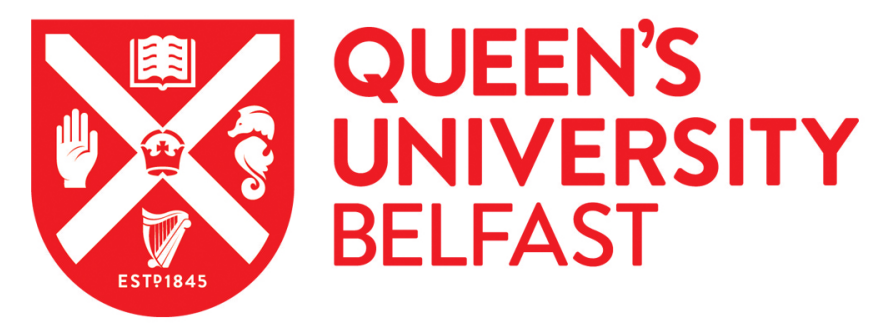

\title{
Laser-accelerated ion beam diagnostics with TOF detectors for the ELIMED beam line
}

Milluzzo, G., Scuderi, V., Amico, A. G., Borghesi, M., Cirrone, G. A. P., Cuttone, G., Napoli, M. D., Doria, D., Dostal, J., Larosa, G., Leanza, R., Margarone, D., Petringa, G., Pipek, J., Romagnani, L., Romano, F., Schillaci, F., \& Velyhan, A. (2017). Laser-accelerated ion beam diagnostics with TOF detectors for the ELIMED beam line. Journal of Instrumentation, 12(2), [C02025]. https://doi.org/10.1088/1748-0221/12/02/C02025

Published in:

Journal of Instrumentation

Document Version:

Peer reviewed version

Queen's University Belfast - Research Portal:

Link to publication record in Queen's University Belfast Research Portal

Publisher rights

(c) 2017 IOP.

This work is made available online in accordance with the publisher's policies. Please refer to any applicable terms of use of the publisher.

\section{General rights}

Copyright for the publications made accessible via the Queen's University Belfast Research Portal is retained by the author(s) and / or other copyright owners and it is a condition of accessing these publications that users recognise and abide by the legal requirements associated with these rights.

Take down policy

The Research Portal is Queen's institutional repository that provides access to Queen's research output. Every effort has been made to ensure that content in the Research Portal does not infringe any person's rights, or applicable UK laws. If you discover content in the Research Portal that you believe breaches copyright or violates any law, please contact openaccess@qub.ac.uk. 


\title{
Laser-accelerated ion beam diagnostics with TOF detectors for the ELIMED beam line
}

\author{
G. Milluzzo ${ }^{a, b, 1}$, V. Scuderi ${ }^{a, c}$, A.G. Amico ${ }^{a}$, M. Borghesi ${ }^{d}$, G.A.P. Cirrone ${ }^{a}$, G. Cuttone $^{a}$, \\ M. De Napoli ${ }^{e}$, D. Doria ${ }^{d}$, J. Dostal ${ }^{f}$, G. Larosa ${ }^{a}$, R. Leanza ${ }^{a, b}$, D. Margarone ${ }^{c}$, G.

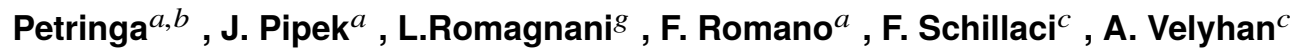 \\ ${ }^{a}$ INFN-LNS, Via S Sofia 62, \\ Catania, Italy \\ ${ }^{b}$ Physics and Astronomy Department, University of Catania \\ Catania, Italy \\ ${ }^{c}$ ELI-Beamline Project, Inst. Physics, ASCR, PALS Center \\ Prague, Czech Republic \\ ${ }^{d}$ School of Mathematics and Physics, Queens University Belfast \\ Belfast, United Kingdom \\ ${ }^{e}$ Istituto Nazionale di Fisica Nucleare, Sezione di Catania, Via S. Sofia 64, I-95123 Catania, Italy \\ $f$ Institute of Plasma Physics ASCR, PALS Laboratory, Prague 182 00, Czech Republic \\ ${ }^{g}$ LULI, Ecole Polytechnique, CNRS, CEA, UPMC, 91128 Palaiseau, France
}

E-mail: giuliana.milluzzo@lns.infn.it

ABSTRACT: Laser-accelerated ion beams could represent the future of particle acceleration in several multidisciplinary applications, as for instance medical physics, hadrontherapy and imaging field, being a concrete alternative to old paradigm of acceleration, characterized by huge and complex machines. In this framework, following on from the ELIMED collaboration, launched in 2012 between INFN-LNS and ELI-Beamlines, in 2014 a three-years contract has been signed between the two institutions for the design and the development of a complete transport beam-line for high-energy ion beams (up to $60 \mathrm{MeV}$ ) coupled with innovative diagnostics and in-air dosimetry devices. The beam-line will be installed at the ELI-Beamlines facility and will be available for users. The measurement of the beam characteristics, such as energy spectra, angular distributions and dose-rate is mandatory to optimize the transport as well as the beam delivery at the irradiation point. In order to achieve this purpose, the development of appropriate on-line diagnostics devices capable to detect high-pulsed beams with high accuracy, represents a crucial point in the ELIMED beamline development. The diagnostics solution, based on the use of silicon carbide ( $\mathrm{SiC})$ and diamond detectors using TOF technique, will be presented together with the preliminary results obtained with laser-accelerated proton beams.

KEYwORDs: high energy laser-driven protons; Diagnostics; Time Of Flight.

ARXIV EPRINT: 1234.56789

${ }^{1}$ Corresponding author. 


\section{Contents}

1 Introduction 1

2 The ELIMED approach $\quad 2$

3 Diagnostics system 4

4 Tests with laser-driven proton beams 5

5 Conclusions $\quad 7$

$\begin{array}{lll}6 & \text { Acknowledgments } & 7\end{array}$

\section{Introduction}

As it is well known, proton and heavy ion radiotherapy for cancer treatment represent a powerful tool allowing the irradiation of tumors with a higher spatial selectivity than the conventional radiotherapy based on photons $[1,2]$. Proton and ion beams with sufficient energy to penetrate the human tissue, are typically accelerated from huge and expensive cyclotrons and synchrotrons. The complexity and the high maintenance costs of the accelerating machines often limit the worldwide spread of hadrontherapy centers, leading to consider innovative and alternative particle acceleration mechanism based machine [3, 4]. In this framework, a growing interest of the scientific community has been addressed to the study of short pulsed high power laser interaction with solid matter as an alternative proton and ion source for fundamental research as well as for multidisciplinary applications, including the medical one [5]. For the first time in 1957, Veksler introduced the concept of "coherent acceleration" [6], indicating the proportionality between the acceleration field for each particle with the number of particles being accelerated, in contrast with the conventional particle acceleration technique. This new concept can be considered as the starting point for the laser-based acceleration field. A high power laser interacting with solid foil, releases instantly its energy in a $\mu \mathrm{m}$ small spot creating a state of ion-electron plasma on the surface. Strong electric fields are generated by the collective displacement of a large number of electrons, accelerating protons and ions until charge neutrality is restored, together with electrons in a ballistic way. According to the laser characteristics, as the intensity, the energy on target and the time duration (ps-fs), different acceleration regimes can be distinguished so far as for instance target normal sheath acceleration (TNSA) [7-9], the radiation pressure acceleration (RPA) [10] and the Break-out afterburner acceleration [11]. The TNSA mechanism, dominant for the intensity regime above $10^{18}$ $\mathrm{W} / \mathrm{cm}^{2}$, has been widely investigated both experimentally and theoretically as reported in literature $[6,12]$. Nevertheless in this regime, proton and ion beams accelerated from laser-target interaction show some peculiar non-conventional characteristics, as the short pulse duration of the order of 
0.1-1 ns, the high peak current up to $10^{12} \mathrm{p} / \mathrm{bunch}$, the broad energy and angular distribution and the poor shot-to-shot reproducibility [6]. As a consequence, in order to use laser-driven beams for different kind of applications, like the hadrontherapy, a complete system suitable to transport, select and control the particle beam from the source to the irradiation point, has to be developed. This is the main goal of several recent projects as the LIBRA (Laser Induced Beams of Radiation and their Applications) project at the Queen's University of Belfast (UK) [13] and OnCoOPtics [14] (High-Intensity Lasersfor Radiooncology), a cooperation between the centers of innovation OncoRay in Dresden and the Helmholtz-Zentrum Dresden-Rossendorf (HZDR). In this framework, the collaboration, born in the 2012, between the Laboratori Nazionali del Sud (LNS-INFN) and ASCR-FZU (Institute of Physics of the Czech Academy of Science) [15] in charge for the installation of the ELI-Beamlines facility in Prague lead to the signature of a three-years contract between the two institutions. The contract main aim is the realization of a whole beamline devoted to transport, handling and dosimetry of the optically accelerated ions for multidisciplinary applications which will be generated from the PW laser, available in the next years in Prague[16]. LNS-INFN is in charge of development, test and installation of all the elements composing the ELIMED beamline which will be installed along the ELIMAIA (ELI Multidisciplinary Applications of laserIon Acceleration) beamline, devoted to the ion acceleration at ELI-Beamlines, by the end of 2017. In this contribution, a general overview of the ELIMED beamline with particular attention to the online diagnostics system, developed to measure the ion energy spectrum and fluence pulse to pulse, will be given.

\section{The ELIMED approach}

The general idea of the ELIMED beamline, entirely developed by the INFN-LNS, is to obtain a stable and reproducible beam at the irradiation point, in spite of the intrinsic shot-to-shot instability, developing specific transport elements, suitable to collect and select the broad energy distribution beam, and appropriate detectors allowing to measure the beam energy spectrum and the absolute dose delivered per pulse [17, 18]. The ELIMED beamline solution is a modular system, composed by three main components: a) collection and diagnostics section, b) transport and energy selection section and c) in-air dosimetry section. Due to the wide angular divergence that can reach $\pm 20^{\circ}$, to collect the largest number of protons a system of five permanent magnet quadrupoles (PMQs) allowing to focus protons up to an energy of $60 \mathrm{MeV}$, has been designed and developed. The PMQs are based on a hybrid Halbach array [19] in order to maximize the magnetic field strength, quality and uniformity by keeping the system as compact as possible. The focus for a specific energy and species is obtained changing the relative distance between each of the PMQs.

In order to improve the transmission efficiency and to optimize the matching with selection system acceptance, the PMQs system is placed downstream the target at about $50 \mathrm{~mm}$ from it. After the quadrupoles system, a diagnostics device will be placed. It will consist of a diamond detector used in TOF configuration and will be described in details in the next section. The measurement of the energy spectrum and number of particles after the PMQs focusing system is indeed a crucial information, allowing the possibility to enhance the transmission and the acceptance for the second transport element: the energy selector system (ESS). The ESS will be composed of four tunable resistive magnets allowing to vary the current and then the magnetic field depending on the ion 


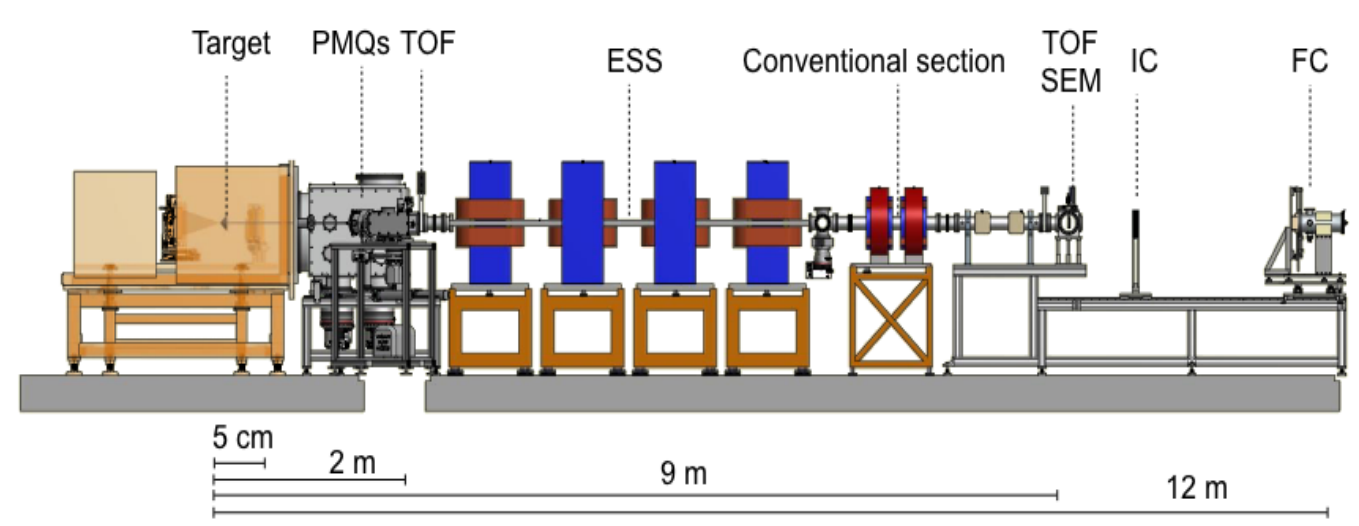

Figure 1. Scheme of the ELIMED beam line showing all the components: the quadrupoles system (PMQs), the Time Of Flight detectors (TOF), the energy selector system (ESS), the conventional part, the secondary emission monitor (SEM), the ionization chamber (IC) and the Faraday Cup (FC). The distances from the target for the first quadrupole, the TOF detector and the final in-air section are reported.

species and the energy to be selected. It will be based on a resistive coil system with alternating field, with a maximum reachable magnetic field of 1.2 Tesla. The output beam is then a beam nearly monochromatic with a maximum energy spread of $5 \%$ for $60 \mathrm{MeV}$ protons. The beam coming out from this first section of the beamline (PMQs+ESS) will have characteristics similar to the conventional beams and, hence, easy to transport and shape with conventional magnetic lenses, such as resistive quadrupoles and steerers. These will be placed in the last part of the in-vacuum beam-line as shown in figure 1. A second TOF detector will be placed after the conventional elements, at about $9 \mathrm{~m}$ from the target, in order to perform the energy and fluence measurement of the already-selected beam and give crucial information for the dosimetry devices placed in-air. A $50 \mu \mathrm{m}$ kapton window divides the in vacuum transport section (PMQs+ESS) from the in-air dosimetry section. In medical applications, such as radiotherapy/hadrontherapy, according to the Code of Practice for Dosimetry IAEA TRS- 398 of 2006 [20], a 5\% accuracy between the prescribed dose and the delivered dose must be reached in order not to compromise the treatment and, as a consequence, the patient's health. The choice of the dosimetry devices has been, then, a critical point during the ELIMED design. The extremely high dose-rate of laser-driven ion beams does not allow the use of dose-rate dependent dosimetry devices as well as conventional ones, as the ionization chamber. For this reason, a Faraday Cup (FC), able to collect the charge in a dose-rate independent way, has been chosen as absolute dosimeter at the end of beamline, while a multi-gap transmission ionization chamber will be employed as relative dosimeter, to be cross-calibrated with respect to the FC. Both dosimetry devices have peculiar geometries, allowing to increase the charge collection efficiency and correct for the gas recombination effect due to the huge particle fluence, respectively [17]. A secondary emission monitor (SEM) will be also used as relative dosimeter, giving information on the particle current. 
Monte Carlo Geant4 simulations [21, 22] of the whole ELIMED beamline have been also carried out, as support for the design of the single elements composing the beamline and to more rigorously evaluate the transport of the beam through the magnetic elements. Moreover, the Geant 4 simulation will be extremely important to improve the beam transport along the in-air section, where an homogeneous beam and a flat profile is required for samples irradiation. The application reproducing the whole ELIMED beamline will be also supplied to ELI-Beamline facility, and it will become together with the ELIMED beamline, a suitable user-friendly tool for all the users that will perform experiments at ELIMAIA.

\section{Diagnostics system}

As it has been already underlined in section 1, laser-driven proton beam characteristics are very far from the conventional ones and, as a consequence, the detection of such high-pulsed beams cannot be performed using traditional particle detectors. In particular, the requirements needed to measure the energy and the flux of laser-driven beams are: radiation hardness ( $\left.>10^{12} \mathrm{p} / \mathrm{bunch}\right)$, fast charge collection, due to the intense proton pulse duration of about $10 \mathrm{~ns}$ and good signal-to-noise ratio, due to the huge electromagnetic pulse (EMP) which characterizes the laser environment [23].

Moreover, considering the high laser repetition rate $(1-10 \mathrm{~Hz})$ at ELIMAIA, the use of detectors able to give online shot-to-shot information of the beam parameters, as energy and intensity, is fundamental in order to monitor and control continuously the beam for applications. A good time resolution is also required to disentangle the different components of the incoming beams, to discriminate the particle species and reconstruct the correspondent energy.

Typically, electrons, protons and ions are accelerated by laser-target interaction and a time evolution signal of all the beam particles produced, i.e. a TOF signal, can be acquired using detectors such as Faraday Cups and ion collectors. Nevertheless, the low time and energy resolution of these detectors limit the time separation of the different accelerated species or/and charge states, required for the identification and the energy reconstruction, particularly, for high energy ( $>10 \mathrm{MeV})$ proton beams.

For all these reasons, $\mathrm{SiC}$ and diamond detectors, offering all these advantageous properties [2426], are the most appropriate for high energy proton beam diagnostics along the ELIMED beamline. In particular, two different types of Chemical Vapour Deposition (CVD) diamond detectors, supplied by the CIVIDEC Instrumentation company [27], have been chosen for laser-accelerated beam diagnostics at ELIMAIA: a $100 \mu \mathrm{m}$ thickness polycrystalline (pCVD) high radiation detector, specifically designed for high intensity loss measurements and a $500 \mu \mathrm{m}$ thickness single crystal diamond detector (sCVD). The detector housing is made of epoxy loaded FR4, gold metalized and completely RF shielded. The whole PCB is contained in an Aluminium box with size $55 \mathrm{~mm} \times 55$ $\mathrm{mm} \times 15 \mathrm{~mm}$ with extra RF shielding, useful for the EMP attenuation. Detector housings have been provided with window apertures of $2 \mathrm{~mm}$ diameter for the pCVD and $3 \mathrm{~mm}$ diameter for the sCVD. The operational characteristics of these two detectors, in terms of intrinsic rise time and response, have been tested using a a ${ }^{241} \mathrm{Am} \alpha$ particle source facing both detectors in-vacuum. The $\alpha$ particle signal has been stored by a $2 \mathrm{GHz}$ Le Croy digital oscilloscope with a $50 \Omega$ load impedance by using a CIVIDEC C2 Broadband Amplifier, and both detectors were biased using a CAEN DT1471HET power supply. The signal amplitude dependence from the applied voltage has been measured for 
the $\mathrm{SCVD}$, as shown in figure $2 \mathrm{a}$. As expected, the signal amplitude increases with the voltage, ranging from 250 Volt to 400 Volt, the latter being the operational value suggested by the company. For such voltage, a rise time of less than $2 \mathrm{~ns}$ has been measured, as shown in the insert of figure 2a. The $\alpha$ signal acquired applying a positive voltage of $200 \mathrm{~V}$ to the $\mathrm{pCVD}$ is also shown in figure $2 \mathrm{~b}$. A rise time of less than $400 \mathrm{ps}$ has been measured with this detector (insert figure $2 \mathrm{~b}$ ). The results, in agreement with the specification given by the CIVIDEC, show as expected, a faster response for the thin $\mathrm{pCVD}$ with respect to the thick sCVD diamond detector.
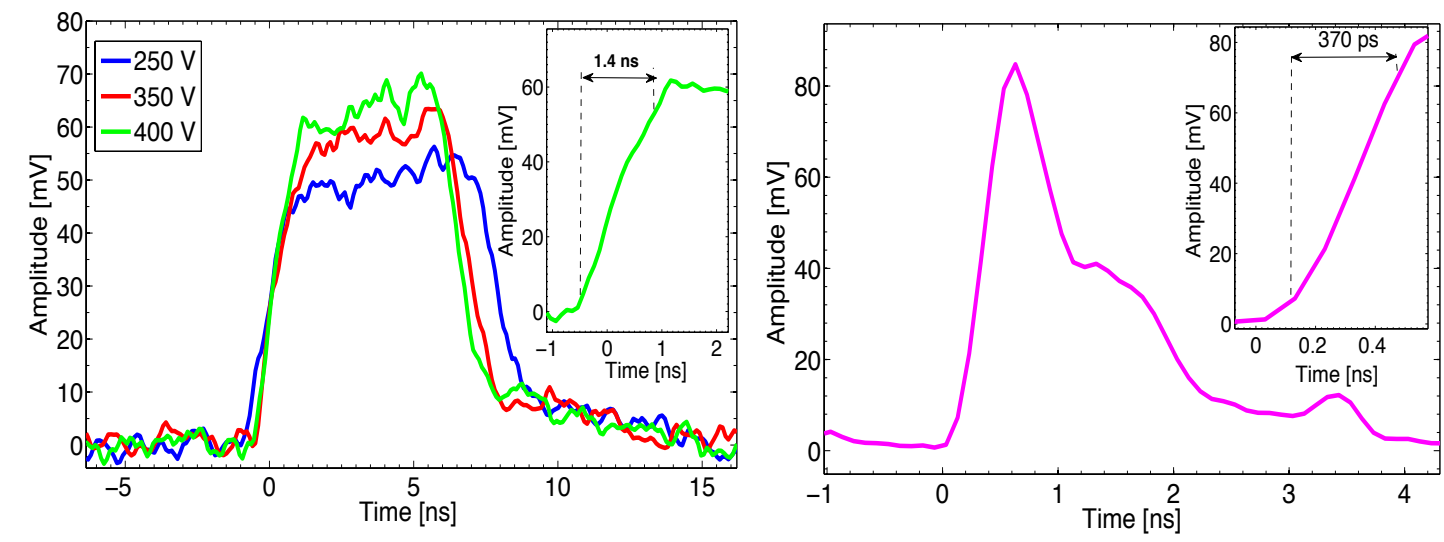

Figure 2. a) $\alpha$ signal acquired by the sCVD detectors with $250 \mathrm{~V}$ (blue), 350 (red) and 400 (green) applied Bias voltage. The insert in the top-right shows the rise time of the signal with 400 Volt Bias voltage. b) $\alpha$ signal acquired by the pCVD detectors with $200 \mathrm{~V}$ applied Bias voltage and rise time of the signal (insert in the top-right).

Also $\mathrm{SiC}$ detectors, used as prototypes for the laser-accelerated beams diagnostics, show similar time and energy response as reported in [28] and will be employed as complementary detectors, particularly in the preliminary phase of the ELIMED installation. As mentioned above, the detectors will be employed using Time Of Flight (TOF) technique and will be placed at two different distances from the target, giving crucial information for the particle transport. The signal will be directly acquired using fast oscilloscopes, connected with a centralized control system, in order to register the signal and perform the preliminary analysis for the on-line beam monitoring.

\section{Tests with laser-driven proton beams}

The pCVD and the sCVD have been recently tested with laser-driven proton beams with the Petawatt VULCAN laser at RAL laser facility. The VULCAN laser is a neodymium glass laser operating at a central wavelength of $1053 \mathrm{~nm}$ and delivering $500 \mathrm{~J}$ on target in a short pulse of about $500 \mathrm{fs}$ [29]. The pCVD and the sCVD have been used to detect the accelerated beams in the backward direction at $1.22 \mathrm{~m}$ from the target and at about $11^{\circ}$ with respect to the target normal axis. A $25 \mu \mathrm{m}$ thick aluminium target was used, inducing the particle acceleration. Figure 3 shows the typical time evolution signal acquired for one shot with the pCVD and registered with a $2.5 \mathrm{GHz}$ digital scope. The first peak appearing at the beginning of time axis corresponds to the so-called photo-peak, arising from UV, X-rays and fast electrons emitted from the target, and fixes the starting point for the acquisition, i.e. the trigger signal. The low sensitivity of thin diamond detector to the light 
reflects in a narrow photo-peak; this is another important advantage for high energy laser-driven beam detection, allowing to discriminate the photo-peak from the high-energy protons characterized by short TOF, especially at short flight path as in the case shown in figure 3 .

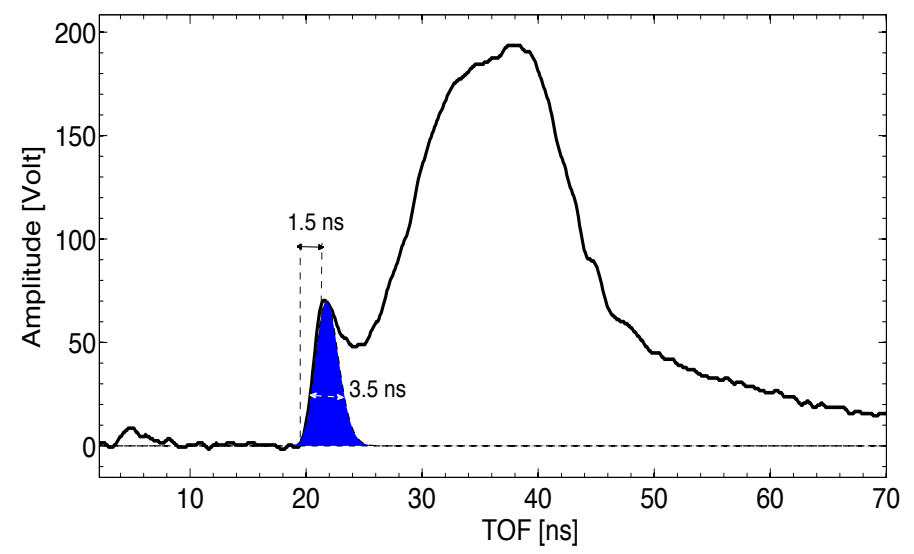

Figure 3. Total signal detected by the pCVD placed at $1.22 \mathrm{~m}$ from the target.

In spite of the short flight path $(1.22 \mathrm{~m})$, two time-separated peaks can be easily disentangled in the TOF signal, shown in figure 3, corresponding respectively to the fast accelerated proton component, about $20 \mathrm{MeV}$, and the slow ion contribution. As indicated in figure 3, a fitting procedure on the peak arising from the fast proton contribution has been performed using the wellknown Maxwell-Boltzmann shifted functions [24], in order to investigate the time response of the detector with such high energy pulsed beams. A rise time of $1.5 \mathrm{~ns}$ and a FWHM of about $3.5 \mathrm{~ns}$ have been measured, showing the fast response of the $\mathrm{PCVD}$, which represents a promising result, in view of the expected $60 \mathrm{MeV}$ protons at ELIMAIA.

The good time resolution allows to distinguish the fast proton component of the beam from the photo-peak as well as from the slow ion contribution also at a short distance from the target. The broad ion contribution (second peak), due to the acceleration of light ions as for instance carbon and oxygen, typically present as contaminants in the target, overlapping with the low-energy proton contribution, makes more difficult the signal deconvolution procedure in this time region, not allowing an easy proton energy spectrum reconstruction.

However, for multidisciplinary applications, especially medical ones, an important improvement can be achieved by developing novel target material, capable of producing only protons (without contamination). For this reason a recent experimental campaign using the ELISE (experiments on laser interaction with solid hydrogen) target system has been performed at PALS laser facility in Prague by using pure cryogenic hydrogen target with an alternative approach that provides a continuous flow of thin laminar targets [30]. This sophisticated target system was used for the first time with the 2 TW PALS laser, characterized by an energy on target of about 600 Joule and a time pulse of $300 \mathrm{ps}$. In this experiment a $21 \mu \mathrm{m}$ thickness $2 \times 2 \mathrm{~mm}^{2} \mathrm{SiC}$ was placed at $0^{\circ}$ at 106 $\mathrm{cm}$ in forward direction to detect the generated protons. A voltage of 150 Volt was applied to the detector to deplete a thickness of about $5 \mu \mathrm{m}$ [31] and $4.8 \mu \mathrm{m}$ thick aluminium absorber was used in order to stop protons up to $450 \mathrm{KeV}$. Figure 4 shows a typical TOF signal registered during a shot with the $\mathrm{SiC}$ detector. In this case, no ions were accelerated from the target and the total signal 
registered is the convolution of different proton populations with different emission energies.

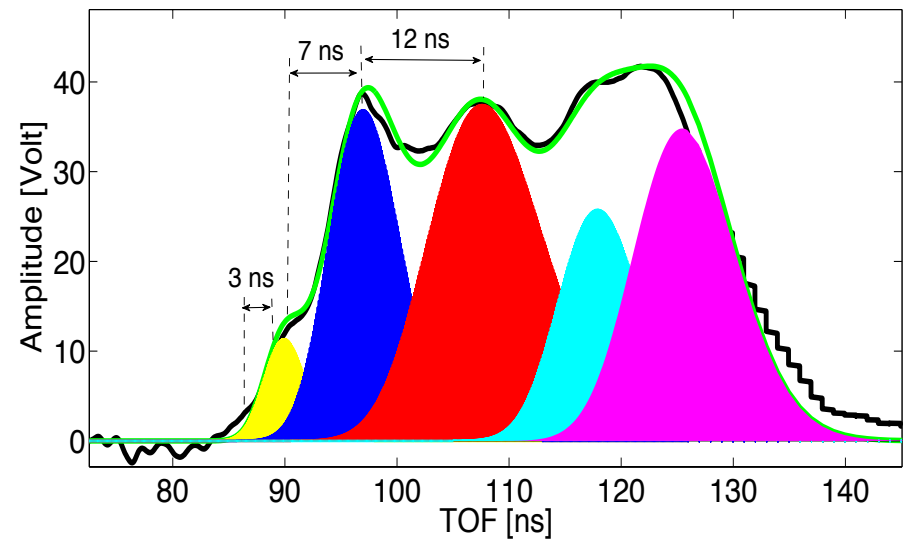

Figure 4. Total TOF signal acquired with $\mathrm{SiC}$ detector placed at 1.06 from the target. The five identified proton distributions are reported with different colours in the plot. The rise time and the time distance between the first and the second peak and the second and the third peak are reported, demonstrating the fast response of the detector

Five different proton contributions have been identified, as shown in figure 4, by performing a deconvolution procedure of the signal with the Maxwell Boltzmann shifted functions, corresponding to an energy ranging from $450 \mathrm{KeV}$ up to $1 \mathrm{MeV}$. A rise time of $3 \mathrm{~ns}$ has been measured, as indicated in figure 4, showing how this kind of diagnostics using TOF method, can represent a powerful tool for the on-line detection of the expected high energy proton beams at ELIMAIA.

\section{Conclusions}

The results reported in the last sections show how a TOF technique, consisting on the particle Time of Flight measurement for laser-driven beam diagnostics, represents one of the most appropriate method for particle energy spectrum and flux reconstruction in this kind of environment. Moreover the use of such devices as diamond and $\mathrm{SiC}$ detectors described above and characterized by a good time and, as a consequence, energy resolution, is a strong requirement in view of the expected $60 \mathrm{MeV}$ proton beams at ELIMAIA. A good time resolution of the diagnostics system along the ELIMED beamline assures the accurate reconstruction of the proton energy spectrum and fluence also at short flight path (just $2 \mathrm{~m}$ after the PMQs system), allowing the optimization of the proton transport through the energy selector system, in terms of transmission and energy spread at the end of the beamline. Furthermore the on-line monitoring of the beam parameters, possible with TOF detectors, allows to tune laser and transport parameters according to the required output beam in the in-air final section, assuring shot-to-shot controllable beams, necessary for application purpose.

\section{Acknowledgments}

This work has been performed within the ELIMED activities supported by the V committee of INFN (Italian Institute for Nuclear Physics), the MIUR (Italian Ministry of Education,Research and University) and by the ELI-Beamlines Contract no S14-187 under CZ. 02.1.01/0.0/0.0/15008/0000162 
through the European Regional Development Fund. We would like to kindly thank the PALS and the RAL staff for their essential support during the performed experiments.

\section{References}

[1] G. Cuttone et al., CATANA protontherapy facility: The state of art of clinical and dosimetric experience. Eur. Phys. J. Plus 2011, 126, 17.

[2] D. Schulz-Ertner, O. Jakel, W. Schlegel, Radiation therapy with charged particles. Semin. Radiat. Oncol. 2006, 16, 249-259.

[3] U.R. Amaldi, S. Bonomi, M. Braccini, A. Crescenti, M. Degiovanni, A. Garlasché, G. Garonna, C. Magrin, P. Mellace, G. Pearce et al. Accelerators for hadron therapy: From lawrence cyclotrons to linacs. Nucl. Instrum. Methods A 2010, 620, 563-577.

[4] J.S. Loeffler \& M. Durante, Charged particle therapy-optimization, challenges and future directions. Nat. Rev. Clin. Oncol. 10, 411-424 (2013).

[5] C.M Ma, R.L. Maughan, C. Orton, Med. Phys. 2006, 33, 571-573.

[6] A. Macchi et al 2013 Rev. Mod. Phys. 85751

[7] S C Wilks et al 2001 Phys. Plasmas 8542

[8] S.P. Hatchett et al., Physics of Plasmas 7 (2000) 2076.

[9] J. Badziak, Opto-Electronics Review 15 (2007) 1.

[10] T. Esirkepov, M. Borghesi, S.V. Bulanov, G. Mourou, T. Tajima, Physical Review Letters (2004) 92, http://dx.doi.org/10.1103/PhysRevLett.92.175003.

[11] L. Yin, B.J. Albright, K.J. Bowers, D. Jung, J.C. Fernandez, B.M. Hegelich, Three-dimensional dynamics of breakout afterburner ion acceleration using high-contrast short-pulse laser and nanoscale targets. Phys. Rev. Lett. 2011, 107, doi:10.1103/PhysRevLett.107.045003.

[12] K. Zeil, S.D. Kraft, S. Bock, M. Bussmann, T.E. Cowan, T. Kluge, J. Metzkes, T. Richter, R. Sauerbrey, U. Schramm, The scaling of proton energies in ultrashort pulse laser plasma acceleration. New J. Phys. 2010, 12, doi:10.1088/1367-2630/12/4/045015.

[13] LIBRA http://www.qub.ac.uk/sites/LIBRA/Resources/.

[14] onCOOPtics http://www.oncoray.de/?id=22?.

[15] D. Margarone, G.A.P. Cirrone, G. Cuttone, G. Korn, AIP Conference Proceedings 1546 (2013) 1.

[16] G.A.P. Cirrone et al. Transport and dosimetric solutions for the ELIMED laser-driven beam line. Nucl. Instrum. 375 Methods Phys. Res. Press 2015, doi:10.1016/j.nima.2015.02.019.

[17] F. Romano et al., The ELIMED transport and dosimetry beamline for laser-driven ion beams , Nuclear Instruments and Methods in Physics Research A 829(2016)153-158

[18] G.A.P. Cirrone et al., Design and Status of the ELIMED Beam Line for Laser-Driven Ion Beams, Appl. Sci. 2015, 5, 427-445; doi:10.3390/app5030427

[19] F. Schillaci et al., Design of a large acceptance, high efficiency energy selection system for the ELIMAIA beam-line, Journal of Instrumentation 11(08):P08022-P08022 ů August 2016 DOI: 10.1088/1748-0221/11/08/P08022 
[20] Absorbed Dose Determination in External Beam Radiotherapy: An International Code of Practice for Dosimetry based on Standards of Absorbed Dose to Water,

www-pub.iaea.org/MTCD/publications/PDF/TRS398_ scr.pdf

[21] S. Agostinelli et al., Geant4-a simulation toolkit, NIMA 506(3) 250-303 (2003)

[22] J. Allison et al., Recent development in Geant4, NIMA Volume 835, 1 November 2016, Pages 186-225 http://dx.doi.org/10.1016/j.nima.2016.06.125

[23] M. De Marco et al., Journal of Physics: Conference Series 508 (2014) 012007.

[24] D. Margarone et al., Full characterization of laser-accelerated ion beams using Faraday cup, silicon carbide, and single-crystal diamond detectors , J. Appl. Phys. 109, 103302 (2011); doi:10.1063/1.3585871

[25] M. Marinelli, E. Milani, G. Prestopino, C. Verona et al., Analysis of laser-generated plasma ionizing radiation by synthetic single crystal diamond detectors , Applied Surface Science 272 (2013) 104-108

[26] G. Bertuccio et al., Silicon carbide detector for laser-generated plasma radiation, Applied Surface Science 272 (2013) 128-131

[27] CIVIDEC https://cividec.at

[28] M. De Napoli et al., Light ions response of silicon carbide detectors, Nuclear Instruments and Methods in Physics Research A 572 (2007) 831-838

[29] C.N Danson et al., Nuclear Fusion, Volume 44, Number 12 http://dx.doi.org/10.1088/0029-5515/44/12/S15

[30] D. Margarone et al., Proton Acceleration Driven by a Nanosecond Laser from a Cryogenic Thin Solid-Hydrogen Ribbon, PHYSICAL REVIEW X 6, 041030 (2016)

[31] M. De Napoli et al., Dopant concentration dependence of the response of SiC Schottky diodes to light ions, Nuclear Instruments and Methods in Physics Research A 600 (2009) 618?623 